\title{
Influence of Finishing/Polishing Procedures on the Surface Texture of Two Resin Composites
}

\author{
Atsushi Kameyama ${ }^{1, *}$, Taeko Nakazawa ${ }^{1}$, Akiko Haruyama $^{1}$, Chikahiro Haruyama ${ }^{1}$, \\ Makoto Hosaka ${ }^{1,2}$ and Yoshito Hirai ${ }^{1}$ \\ ${ }^{I}$ Department of Operative Dentistry, Tokyo Dental College, Chiba, Japan and ${ }^{2}$ Department of Oral Hygiene, Chiba \\ College of Health Science, Chiba, Japan
}

\begin{abstract}
This study compared surface roughness and gloss produced by different finishing/polishing procedures for two resin composites, Clearfil AP-X (AP-X) and Estelite $\Sigma$ (ES). A total of 70 composite discs ( $=35$ for each resin composite) were prepared and divided at random into seven finishing/polishing groups ( $n=5)$ : glass-pressed control; using a super-fine-grit diamond bur (SF); using CompoMaster (CM) after SF-finishing (SF+CM); using White Point (WP) after SFfinishing (SF+WP); using CM after SF+WP-finishing (SF+WP+CM); using Stainbuster (SB) after SF-finishing (SF+SB); and using $\mathrm{CM}$ after $\mathrm{SF}+\mathrm{SB}$-finishing $(\mathrm{SF}+\mathrm{SB}+\mathrm{CM})$. After the finishing/polishing procedures, average surface roughness $\left(R_{\mathrm{a}}\right)$ and surface gloss $\left(\mathrm{Gs}\left(60^{\circ}\right)\right)$ of all specimens were assessed with a surface profilometer and specimen gloss meter, respectively. Glass-pressed controls for both AP-X and ES composites showed the best surface finish in terms of both $R_{\mathrm{a}}$ and $\mathrm{Gs}\left(60^{\circ}\right)$. SF-finishing produced the roughest surface and led to almost complete loss of gloss. While additional polishing with CM reduced $R_{\mathrm{a}}$ and increased Gs $\left(60^{\circ}\right)$, the additional finishing effect of WP or SB between SF-finishing and $\mathrm{CM}$-polishing was not found for either AP-X or ES.
\end{abstract}

Key Words: Surface roughness, Resin composite, Finishing, Polishing, Surface gloss.

\section{INTRODUCTION}

Resin composite restoration currently achieves esthetically pleasing and natural-looking results through the use of nanoparticle-sized small fillers, variations of color, and newly developed restorative techniques such as the layering technique (Peyton PPAD 2002) [1]. To ensure the longevity of these esthetic composite restorations some aspects must be taken in consideration, such as: strong and stable adhesion to teeth, techniques involving gap-free filling, authentic polymerization, functional and anatomical contouring with over-or-less filling, and smooth and glossy polishing (Jung OD 2007) [2].

Finishing and polishing processes, including the removal of excess-filled resin composite, shaping, contouring, and smoothing of the restoration, can affect many aspects of the final restoration, such as surface staining, plaque accumulation, gingival irritation, and wear characteristics of the composite (Murchison Fundamentals of Operative Dentistry 2006) [3] (Shintani DM 1985) [4] (Lu JERD 2005) [5] (Larato JPD 1972) [6]. A wide variety of finishing and polishing instruments are commercially available to the clinician. As initial finishing, it has been advocate the use a fine-grit diamond or multi-fluted tungsten carbide bur to remove the excess-filled composite and shape the anatomical contouring; and silicone-based points and/or abrasive discs for final polish (Jung OD 2003) [7] (Roeder OD 2000) [8] (Barbosa BDJ 2005) [9]. The additional use of an aluminum-oxide abrasive point may be applied for second finishing between first finishing and final polishing.

*Address correspondence to this author at the Department of Operative Dentistry, Tokyo Dental College, Chiba, Japan;

E-mail: kameyama@tdc.ac.jp
The resin composite Estelite $\Sigma$ (Tokuyama Dental Corp., Tsukuba, Japan), with submicron particles of spherical organic filler produced by the sol-gel method, has recently been developed. Lu et al. reported that this composite has similar properties to nano-filled, two micro-filled and two micro-hybrid composites (Lu OD 2006) [10]. However, its surface texture after finishing/polishing has yet to be reported. The present study compared the average surface roughness and surface gloss of one spherical-filled submicron composite (Estelite $\Sigma$ ) and one micro-hybrid composite (Clearfil AP-X, Kuraray Medical, Osaka, Japan) as produced by different finishing/polishing procedures. Furthermore, interactions between the average surface roughness and gloss were also evaluated. The null hypotheses tested in this study were that there is no significant difference in surface texture 1) among the different finishing/polishing procedures, 2) between the two resin composites, and 3) there is no significant interaction between surface roughness and surface gloss.

\section{MATERIALS AND METHODS}

The resin composites used in this study were Clearfil AP$\mathrm{X}(\mathrm{AP}-\mathrm{X})$ and Estelite $\Sigma$ (ES; Tokuyama Dental Corp, Tsukuba, Japan), as shown in Table 1. An acrylic ring (9 mm inner hole diameter, $2.5 \mathrm{~mm}$ depth) was filled with resin composite and sandwiched between two glass slides. The filled resin composite was polymerized with a quartztungsten-halogen light-curing unit (New-Light VL-2, GC, Tokyo, Japan) for $30 \mathrm{~s}$ on each side of the specimen. The light-curing unit was adjusted to $700 \mathrm{~mW} / \mathrm{cm}^{2}$, as measured by the curing radiometer (Model 100, Demetron Research Co., Danbury, CT, USA). 
Also shown in Table $\mathbf{1}$ are the details of the rotary polishing instruments used in this study; the Diamond Point FG (SF; Shofu, Kyoto, Japan), the White Point CA (WP; Shofu), the Stainbuster (SB; Danville Materials, CA, USA) and the CompoMaster (CM; Shofu). Thirty-five specimens of each composite (total: 70 specimens) were randomly assigned to one of seven test groups, as shown in Table 2. Surfaces that were pressed by glass slides were considered as control. All other specimens were surfaced with the super-fine-grit finishing diamond bur SF attached to a 1:5 transmitted highspeed contra-angle motor handpiece (KaVo, Biberach, Germany) for $30 \mathrm{~s}$ at $200,000 \mathrm{rpm}$ to simulate the initial finishing of the composite materials.

The SF-abraded surfaces were then further abraded with SB or WP attached to a 1:1 contra-angle motor handpiece $(\mathrm{KaVo})$ for $30 \mathrm{~s}$ at $9,000 \mathrm{rpm}$. Each of the five specimens with SB- or WP-abraded surfaces were then further abraded with $\mathrm{CM}$ attached to a 1:1 contra-angle motor handpiece $(\mathrm{KaVo})$ for $30 \mathrm{~s}$ at 9,000 rpm. Each procedure of finishing and polishing was conducted under a fine water spray.
After the abrasion/polishing procedures, specimens were rinsed under the water spray and air-dried. Surface roughness was measured using a surface profilometer (Handysurf E30A, Tokyo Seimitsu, Tokyo, Japan), with a standard cutoff of $0.8 \mathrm{~mm}$, a transverse length of $0.8 \mathrm{~mm}$, and a stylus speed of $0.6 \mathrm{~mm} / \mathrm{s}$. The surface roughness was measured five times for each specimen, and the average value obtained was defined as $R_{\mathrm{a}}$ (roughness average) of each specimen.

In addition, the surface gloss of each specimen was measured using a precision gloss meter (GM-260, Murakami Color Research Laboratory, Tokyo, Japan) with the light source and detector both set at $60^{\circ}$ to normal. Before measurement, the gloss meter was calibrated to a standard gloss board $\left(\mathrm{Gs}\left(60^{\circ}\right)=92.1 \%\right)$. Each specimen was measured five times and the average value determined.

Additionally, the amount of the abrasive reduction of each specimen was calculated as the difference between the weight of before and after the finishing/polishing procedure which was measured by weight analytical balancer (GR-202, A\&D Co., Ltd., Tokyo, Japan).

Table 1. Resin Composite Materials and Finishing/polishing Instruments Tested in this Study

\begin{tabular}{|c|c|c|c|c|c|}
\hline \multicolumn{6}{|c|}{ Resin Composite } \\
\hline Code & Resin Composite & Shade & Lot \# & Type (Filler Size) & Manufacturer \\
\hline AP-X & Clearfil AP-X & $\mathrm{A} 3$ & $1115 \mathrm{AB}$ & $3.0 \mu \mathrm{m}$ (hybrid) & Kuraray Medical \\
\hline ES & Estelite $\sum$ & A3 & J22116S & $0.2 \mu \mathrm{m}$ (submicron) & Tokuyama Dental \\
\hline \multicolumn{6}{|c|}{ Finishing and Polishing Instrument } \\
\hline Code & Material & Shade & Lot \# & Type & Manufacturer \\
\hline SF & Diamond Point FG & SF114 & 30610 & $25 \mu \mathrm{m}$ diamond & Shofu \\
\hline WP & White Point CA & No. 44 & 0206534 & $20 \mu \mathrm{m}$ alminum oxide & Shofu \\
\hline $\mathrm{SB}$ & Stainbuster & 2504 & 7640 & $17 \mu \mathrm{m}$ zircon oxide fiber & Danville Materials \\
\hline $\mathrm{CM}$ & Compomaster CA & $13 \mathrm{~S}$ & 0106133 & $6 \mu \mathrm{m}$ diamond & Shofu \\
\hline
\end{tabular}

Table 2. Effect of Finishing/Polishing Procedures on Surface Roughness $\left(R_{a}\right)$ of Each Resin Composites (Mean and SD, $\left.\mu m\right)$

\begin{tabular}{|c|c|c|c|}
\hline Composite/Finishing/Polishing Group* & AP-X & Statistics** & ES \\
\hline \hline Control & $0.08(0.02) \mathrm{C}$ & $\mathrm{NS}$ & $0.06(0.01) \mathrm{c}$ \\
\hline $\mathrm{SF}$ & $0.25(0.01) \mathrm{A}$ & $\mathrm{NS}$ & $0.58(0.10) \mathrm{a}$ \\
$\mathrm{SF}+\mathrm{CM}$ & $0.39(0.02) \mathrm{AB}$ & $\mathrm{S}$ & $0.22(0.01) \mathrm{b}$ \\
\hline SF+WP & $0.47(0.07) \mathrm{AB}$ & $\mathrm{NS}$ & $0.51(0.06) \mathrm{a}$ \\
SF+WP+CM & $0.42(0.09) \mathrm{AB}$ & $\mathrm{NS}$ & $0.43(0.08) \mathrm{a}$ \\
\hline $\mathrm{SF}+\mathrm{SB}$ & $0.36(0.02) \mathrm{B}$ & $\mathrm{NS}$ & $0.51(0.17) \mathrm{a}$ \\
SF+SB+CM & $0.41(0.07) \mathrm{AB}$ & $\mathrm{S}$ & $0.25(0.04) \mathrm{b}$ \\
\hline
\end{tabular}

*SF: superfine diamond bur, CM: CompoMaster, WP: White Point CA, SB: Stainbuster.

** Statistically analysis between AP-X and ES $(p=0.05)$.

S: significantly different, NS: no statistically different.

Values with the same letter are not significantly different in same composite $(p<0.05)$. 
The obtained data were used to calculate the mean and standard deviation (SD) for each group, and were statistically analyzed using two-way analysis of variance (ANOVA), with statistical significance set at a $p$-value of 0.05 . The strength of the association between pairs of variables was obtained by the use of Pearson's correlation coefficient. All statistical analyses were performed using StatView 5.0J (SAS Institute, Cary, NC, USA).

\section{RESULTS}

Values for $R_{\mathrm{a}}, \mathrm{Gs}\left(60^{\circ}\right)$ and the amount of abrasive reduction for each test group are summarized in Tables 2-4, respectively. Two-way ANOVA revealed that all of the finishing methods had a significant effect on $R_{\mathrm{a}} \quad(F=52.021$, $p<0.0001)$ and $\operatorname{Gs}\left(60^{\circ}\right)(F=99.303, p<0.0001)$. The composite material had a significant effect on $\mathrm{Gs}\left(60^{\circ}\right)(F=210.552$, $p<0.0001)$ but not on $R_{\mathrm{a}}(F=0.698, p=0.4071)$. An interaction was found between 'finishing methods' and 'composite material' in terms of both $R_{\mathrm{a}}(F=6.804, p<0.0001)$ and $\mathrm{Gs}\left(60^{\circ}\right)$ $(F=16.879, p<0.0001)$. Therefore, one-way ANOVA and post-hoc Tukey's test was additionally performed in both $R_{\mathrm{a}}$ and $\mathrm{Gs}\left(60^{\circ}\right)$ at the $5 \%$ level, respectively.

The glass-pressed controls significantly found the lowest $R_{\mathrm{a}}$ values $(p<0.05)$ and highest gloss values $(p<0.05)$ among all groups in terms of both AP-X and ES. On the other hand, the SF-abraded groups were the highest $R_{\mathrm{a}}$ values in both AP-X and ES.

For AP-X, SF+SB significantly reduced $R_{\mathrm{a}}$ compared to SF only $(p<0.05)$, while no significant difference was noted between SF and SF+WP $(p>0.05)$. On the other hand, for ES, significant differences were not observed between the SF, $\mathrm{SF}+\mathrm{WP}$, and $\mathrm{SF}+\mathrm{SB}$ groups $(p>0.05)$.

On comparison of WP and SB, no significant difference in $R_{\mathrm{a}}$ was found for either the AP-X or ES composite $(p>0.05)$. A tendency toward the reduction of $R_{\mathrm{a}}$ on additional polishing with $\mathrm{CM}$ was noted, but a significant difference was found only with the $\mathrm{SF}+\mathrm{SB}+\mathrm{CM}$ procedure for $\mathrm{ES}$ $(p<0.05)$

The glass-pressed controls in both AP-X and ES significantly found the highest gloss values $(p<0.05)$ among all groups. The SF-abrasion significantly reduced the gloss values in terms of both AP-X and ES. In AP-X, there is no significant difference among six groups except for the control $(p>0.05)$. On the other hand, CM-polishing significantly increased the gloss value $(p<0.05)$.

Overall, the smoother specimen showed a significantly higher gloss value $(r=-0.724, p<0.001)$. Comparing the two

Table 3. Effect of Finishing/Polishing Procedures on Gloss Value $\left(\operatorname{Gs}\left(60^{\circ}\right)\right)$ of Each Resin Composites (Mean and SD, \%)

\begin{tabular}{|c|c|c|c|}
\hline Composite/Finishing/Polishing Group* & AP-X & Statistics** & ES \\
\hline \hline Control & $88.9(3.5) \mathrm{A}$ & $\mathrm{NS}$ & $81.4(4.0) \mathrm{a}$ \\
\hline $\mathrm{SF}$ & $2.3(0.1) \mathrm{B}$ & $\mathrm{NS}$ & $4.7(1.7) \mathrm{e}$ \\
$\mathrm{SF}+\mathrm{CM}$ & $2.7(0.3) \mathrm{B}$ & $\mathrm{S}$ & $32.5(15.9) \mathrm{bcd}$ \\
\hline $\mathrm{SF}+\mathrm{WP}$ & $11.6(2.0) \mathrm{B}$ & $\mathrm{S}$ & $53.6(9.5) \mathrm{d}$ \\
$\mathrm{SF}+\mathrm{WP}+\mathrm{CM}$ & $11.5(18.8) \mathrm{B}$ & $\mathrm{S}$ & $38.6(9.4) \mathrm{cd}$ \\
SF+SB & $5.9(1.3) \mathrm{B}$ & $\mathrm{S}$ & $60.6(8.6) \mathrm{b}$ \\
\hline SF+SB+CM & $3.7(1.3) \mathrm{B}$ & $\mathrm{S}$ & \\
\hline
\end{tabular}

*SF: superfine diamond bur, CM: CompoMaster, WP: White Point CA, SB: Stainbuster

** Statistically analysis between AP-X and ES ( $p=0.05)$

S: significantly different, NS: no statistically different.

Values with the same letter are not significantly different in same composite $(p<0.05)$

Table 4. Effect of Finishing/Polishing Procedures on Abrasive Reduction of Each Resin Composites (Mean and SD, mg)

\begin{tabular}{|c|c|c|c|}
\hline Composite/Finishing/Polishing Group* & AP-X & Statistics** & ES \\
\hline \hline Control & $0.0(0.0)$ & NS & $14.0(0.0)$ \\
\hline SF & $8.6(1.6)$ & NS & $15.1)$ \\
SF+CM & $18.6(0.6)$ & NS & $13.0)$ \\
\hline SF+WP & $11.1(1.7)$ & NS & $16.9(1.7)$ \\
SF+WP+CM & $15.2(2.9)$ & NS & S \\
SF+SB & $11.7(3.3)$ & NS & $18.1(2.2)$ \\
SF+SB+CM & $14.6(2.1)$ & $16.9)$ \\
\hline
\end{tabular}

*SF: superfine diamond bur, CM: CompoMaster, WP: White Point CA, SB: Stainbuster

** Statistically analysis between AP-X and ES ( $\mathrm{p}=0.05$ )

S: significantly different, NS: no statistically different. 
resin composites, ES tended to have a glossier surface than that of AP-X (AP-X: correlation coefficient $r=-0.887$, $p<0.001$, ES: correlation coefficient $r=-0.804, p<0.001$ ).

\section{DISCUSSION}

One of the purposes of this study was to evaluate the effect of seven different procedures on the surface texture of two resin composites. Several previous studies have measured the polyester matrix strip-produced surface as a control, such as the Mylar strip, followed by clinical application (Roeder OD 2000) [8] (Ozgünaltay JOR 2003) [11] (Türkün OD 2004) [12] (Yap JOR 1998) [13]. Almost all of these procedures resulted in a smoother finish than any polished surfaces. Our study also identified the smoothest and shiniest surfaces to be the glass-pressed controls for both the AP-X and ES resin composites. However, these surfaces result in a reduction in hardness or surface discoloration due to insufficient polymerization or a rich content of organic resin binder (Park JOR 2000) [14] (Park JOR 2004) [15] (Baseren JBA 2004) [16]. Therefore, the removal of the outermost composite by finishing/polishing procedures is necessary to produce a wear-resistant, harder, and color stabilized restoration.

In general, finishing procedures are performed with rigid rotary instruments, such as super-fine-grit diamond burs or tungsten carbide finishing burs. The present study used super-fine-grit diamond burs with an average of $30 \mu \mathrm{m}$ diameter diamond particles. These surfaces were visibly very rough, with a loss of shininess, and numerous scratches. These results were similar to those of previous reports (Jung OD 2007) [2] (Roeder OD 2000) [8] (Reis AJD 2002) [17].

We used an abrasive point with aluminum oxide (WP) as one of the secondary finishing instruments. Our results did not reveal an effect of WP on $R_{\mathrm{a}}(p>0.05)$. However, the effect of WP on glossiness was found only in ES $(p<0.05)$. These findings suggest that the abrasion of rough surfaces created by super-fine-grit diamond bur would reduce the roughness, but would not contribute to the removal of scratches.

We also evaluated the effect of SB in comparison with WP. This newly-developed and unique rotary instrument is based on zircon fiber and binding resin. It is mainly used for the removal of stained tooth surfaces, smoothing after scaling, and the abrasion of adhesive resin from enamel surface on removal of an orthodontic bracket. According to the manufacturer's instruction, this instrument can be used for the finishing of a composite surface. Although a significant decrease in $R_{\mathrm{a}}$ was found for AP-X, no significant difference was noted for ES between the $\mathrm{SF}$ and $\mathrm{SF}+\mathrm{SB}$ procedures. This result might be due to the surface undulation, caused by extensive removal by SF in ES, where the rigid SB instrument could not evenly contact the surface, thus leading to the difference of $R_{\mathrm{a}}$. The larger standard deviation (SD) may support this suggestion.

This study also evaluated the influence of second finishing on final polishing by the use of CM. In terms of $R_{\mathrm{a}}$, there was no difference for the same composite between the three finishing groups $\mathrm{SF}+\mathrm{WP}+\mathrm{CM}, \mathrm{SF}+\mathrm{SB}+\mathrm{CM}$, and $\mathrm{SF}+\mathrm{CM}$, but for ES, a difference was observed between $\mathrm{SF}+\mathrm{WP}+\mathrm{CM}$ and $\mathrm{SF}+\mathrm{SB}+\mathrm{CM}$. Gs $\left(60^{\circ}\right)$ also did not show a significant difference between the three finishing groups. Miyazaki et al. reported that $R_{\mathrm{a}} / R_{\max }$ of WP-finished composite surfaces was equal to the \#400-\#600 $\mathrm{SiC}$ paper-ground composite surface (Miyazaki JJCD 2000) [18]. In clinical situations, second finishing with WP or SB should be used only in the anatomical formation as a backup for diamond/carbide finishing. The first hypothesis, there is no significant difference in surface texture among the different finishing/polishing procedures, was therefore rejected.

Although two-way ANOVA clearly found the significant effect of composite material on surface gloss, no significant effect was found on surface roughness. Therefore, the second hypothesis, there is no significant difference in surface texture, was partially accepted. ES tended to be glossier than AP-X when each $R_{\mathrm{a}}$ was the same. These results are probably due to the difference in filler shape. Composed fillers in almost composites are ground glass particles whose morphology is irregular (Lu OD 2006) [10]. On the other hand, the filler shape of ES was spherical, with a narrow range of 0.1 to $0.3 \mu \mathrm{m}$-sized particles. Therefore, ES might reflect the light uniformly with lower diffusion/absorbance than AP-X (Lu OD 2006) [10].

To evaluate the polishing effect, the measurement of surface gloss is effective as an additional parameter to $R_{\mathrm{a}}(\mathrm{Mi}-$ yazaki JJCD 2000) [18] (Stanford JADA 1985) [19] (Ohmura JJCD 1996) [20] (Watanabe QI 2006) [21] (Da Costa JERD 2007) [22]. Therefore, we also evaluated the effect of each finishing/polishing procedure on gloss value, and interactions between the average surface roughness and gloss. A clear relationship was found between $R_{\mathrm{a}}$ and Gs $\left(60^{\circ}\right)$ in each composite. Therefore, the last hypothesis, there is no significant interaction between surface roughness and surface gloss, was rejected.

\section{CONCLUSIONS}

This study compared the average surface roughness and surface gloss of two composites as produced by different finishing/polishing procedures. Furthermore, interactions between the average surface roughness and gloss were also evaluated, and concluded as follows:

1. The effect of finishing/polishing procedures on surface roughness and gloss were found.

2. While no significant difference in surface roughness was found between two composites, the Estelite $\Sigma$ provided the glossier surface than Clearfil AP-X.

3. A clear relationship was found between average roughness and gloss in each composite.

\section{REFERENCES}

[1] Peyton JH. Direct restoration of anterior teeth: review of the clinical technique and case presentation. Pract Proc Aesthet Dent 2002; 14: $203-10$

[2] Jung M, Sehr K, Klimek J. Surface texture of four nanofilled and one hybrid composite after finishing. Oper Dent 2007; 32: 45-52.

[3] Murchison DF, Roeters J, Vargas MA, Chan DCN (2006) Direct anterior restorations In: Summitt JB, Robbins JW, Hilton TJ, Schwartz ES, Ed. Fundamentals of Operative Dentistry: A Contemporary Approach, Third edition, Chicago, Quintessence Publishing 284.

[4] Shintani H, Satou J, Satou N, Hayashihara H, Inoue T. Effects of various finishing methods on staining and accumulation of Streptococcus mutans HS-6 on composite resins. Dent Mater 1985; 1: 225 7. 
[5] Lu H, Roeder LB, Lei L, Powers JM. Effect of surface roughness on stain resistance of dental resin composites. J Esthet Restor Dent 2005; 17: 102-9.

[6] Larato DC. Influence of composite restoration on the gingiva. J Prosthet Dent 1972; 28: 402-4.

[7] Jung M, Voit S, Klimek J. Surface geometry of three packable and one hybrid composite after finishing. Oper Dent 2003; 28: 53-9.

[8] Roeder LB, Tate WH, Powers JM. Effects of finishing and polishing procedures on the surface roughness of packable composites. Oper Dent 2000; 25: 534-43.

[9] Barbosa SH, Zanata RL, Navarro MFL, Nunes OB. Effect of different finishing and polishing techniques on the surface roughness of microfilled, hybrid and packable composite resins Braz Dent $\mathbf{J}$ 2005; 16: 39-44

[10] Lu H, Lee YK, Oguri M, Powers JM. Properties of a dental resin composite with a spherical inorganic filler. Oper Dent 2006; 31: 734-40.

[11] Ozgünaltay G, Yazici AR, Görücü J. Effect of finishing and polishing procedures on the surface roughness of new tooth-coloured restoratives J Oral Rehabil 2003; 30: 218-24.

[12] Türkün LS, Türkün M. The effect of one-step polishing system on the surface roughness of three esthetic resin composite materials. Oper Dent 2004; 29: 203-11.

[13] Yap AUJ, Sau CW, Lye KW. Effects of finishing/polishing time on surface characteristics of tooth-coloured restoratives. J Oral Rehabil 1998; 25: 456-61.

[14] Park SH, Krejci I, Lutz F. Hardness of celluloid strip-finished or polished composite surfaces with time. J Prosthet Dent 2000; 83: 660-3.
[15] Park SH, Noh BD, Ahn HJ, Kim HK. Celluloid strip-finished versus polished composite surface: difference in surface discoloration in microhybrid composites. J Oral Rehabil 2004; 31: 62-6.

[16] Baseren M. Surface roughness of nanofill and nanohybrid composite resin and ormocer-based tooth-colored restorative materials after several finishing and polishing procedures. J Biomater Appl 2004;19: 121-34.

[17] Reis AF, Giannini M, Lovadino JR, dos Santos Dias CT. The effect of six polishing systems on the surface roughness of two packable resin-based composites. Am J Dent 2002; 15: 193-7.

[18] Miyazaki M, Yamada M, Ando S, Onose H, Kawamura H, Asano $\mathrm{N}$, Sonoi S, Asao O. A study on one-step polishing points: Influence of polishing duration and handpiece load on surface texture and light-cured resins. Jpn J Conserv Dent 2000; 43: 1040-9. (In Japanese)

[19] Stanford WB, Fan PL, Wozniak WT, Stanford JW. Effect of finishing on color and gloss of composites with different fillers. J Am Dent Assoc 1985; 110: 211-3.

[20] Ohmura Y. Effect of polishing methods on the surface characteristics of composite resins. Jpn J Conserv Dent 1996; 39: 1271-87. (In Japanese)

[21] Watanabe T, Miyazaki M, Moore BK. Influence of polishing instruments on the surface texture of resin composites. Quintessence Int 2006; 37: 61-7.

[22] Da Costa J, Ferracane J, Paravina RD, Mazur RF, Roeder L. The effect of different polishing systems on surface roughness and glos of various resin composites. J Esthet Restor Dent 2007;19: 214-24.

(C) Kameyama et al.; Licensee Bentham Open.

This is an open access article licensed under the terms of the Creative Commons Attribution Non-Commercial License (http://creativecommons.org/licenses/ by-nc/3.0/) which permits unrestricted, non-commercial use, distribution and reproduction in any medium, provided the work is properly cited. 